\title{
Comparison of collective Thomson scattering signals due to fast ions in ITER scenarios with fusion and auxiliary heating
}

Salewski, Mirko; Asunta, O.; Eriksson, L.-G.; Bindslev, Henrik; Hynönen, V.; Korsholm, Søren Bang; Kurki-Suonio, T.; Leipold, Frank; Meo, Fernando; Michelsen, Poul

Total number of authors:

12

Published in:

Plasma Physics and Controlled Fusion

Link to article, DOI:

$10.1088 / 0741-3335 / 51 / 3 / 035006$

Publication date:

2009

Link back to DTU Orbit

Citation $(A P A)$ :

Salewski, M., Asunta, O., Eriksson, L-G., Bindslev, H., Hynönen, V., Korsholm, S. B., Kurki-Suonio, T., Leipold, F., Meo, F., Michelsen, P., Nielsen, S. K., \& Pedersen, J. R. (2009). Comparison of collective Thomson scattering signals due to fast ions in ITER scenarios with fusion and auxiliary heating. Plasma Physics and Controlled Fusion, 51(3), 035006. https://doi.org/10.1088/0741-3335/51/3/035006

\section{General rights}

Copyright and moral rights for the publications made accessible in the public portal are retained by the authors and/or other copyright owners and it is a condition of accessing publications that users recognise and abide by the legal requirements associated with these rights.

- Users may download and print one copy of any publication from the public portal for the purpose of private study or research.

- You may not further distribute the material or use it for any profit-making activity or commercial gain

- You may freely distribute the URL identifying the publication in the public portal 


\title{
Comparison of Collective Thomson Scattering Signals due to Fast Ions in ITER Scenarios with Fusion and Auxiliary Heating
}

\author{
M Salewski ${ }^{1}$, O Asunta ${ }^{2}$, L-G Eriksson ${ }^{3}$, H Bindslev $^{1}$, V \\ Hynönen $^{2}$, S B Korsholm ${ }^{1}$, T Kurki-Suonio ${ }^{2}$, F Leipold ${ }^{1}$, F \\ Meo $^{1}$, P K Michelsen ${ }^{1}$, S K Nielsen ${ }^{1}$, J Roenby ${ }^{1}$ \\ ${ }^{1}$ Association Euratom - Ris $\varnothing$ National Laboratory for Sustainable Energy, Technical \\ University of Denmark, DK-4000 Roskilde, Denmark \\ ${ }^{2}$ Association Euratom - TEKES, Helsinki University of Technology, FI-02015 TKK, \\ Finland \\ ${ }^{3}$ CEA, IRFM, F-13108 St. Paul lez Durance, France \\ E-mail: mirko.salewski@risoe.dk
}

\begin{abstract}
Auxiliary heating such as neutral beam injection (NBI) and ion cyclotron resonance heating (ICRH) will accelerate ions in ITER up to energies in the $\mathrm{MeV}$ range, i.e. energies which are also typical for alpha particles. Fast ions of any of these populations will elevate the collective Thomson scattering (CTS) signal for the proposed CTS diagnostic in ITER. It is of interest to determine the contributions of these fast ion populations to the CTS signal for large Doppler shifts of the scattered radiation since conclusions can mostly be drawn for the dominant contributor. In this study, fast ion distribution functions are calculated for beam ions with the ASCOT code or for fast ions generated by ICRH with the PION code for a steady-state ITER burning plasma equilibrium. The parameters for the auxiliary heating systems correspond to the design currently foreseen for ITER. The geometry of the CTS system for ITER is chosen such that near perpendicular and near parallel velocity components are resolved. In the investigated ICRH scenario, waves at $50 \mathrm{MHz}$ resonate with tritium at the second harmonic off-axis on the low field side. Effects of a minority heating scheme with ${ }^{3} \mathrm{He}$ are also considered. CTS scattering functions for fast deuterons, fast tritons, fast ${ }^{3} \mathrm{He}$, and the fusion born alphas are presented, revealing that fusion alphas dominate the measurable signal by an order of magnitude or more in the Doppler shift frequency ranges typical for fast ions. Hence the observable CTS signal can mostly be attributed to the alpha population in these frequency ranges. The exceptions are limited regions in space with some non-negligible signal due to beam ions or fast ${ }^{3} \mathrm{He}$ which give rise to about $30 \%$ and $10-20 \%$ of the CTS signal, respectively. In turn, the dominance of the alpha contribution implies that the effects of other fast ion contributions will be difficult to observe by CTS.
\end{abstract}




\section{Introduction}

Collective Thomson scattering (CTS) is a many-sided diagnostic which, among other potentials, has the capability to characterize the fast ion distribution as demonstrated at JET and TEXTOR [1-4]. The measurement of fast ions is especially important as conditions of burning plasmas are approached or reached [5-7]. The ITER plasma will have substantial populations of supra-thermal fast ions in the $\mathrm{MeV}$ range due to fusion reactions and due to auxiliary heating such as neutral beam injection (NBI) and ion cyclotron resonance heating (ICRH). Since ITER will access the burning plasma regime with at least equal amounts of alpha heating and auxiliary heating as the first tokamak, it will be of considerable interest to measure the phase space distribution of confined energetic ions in these burning plasmas. Current theories describing redistribution and loss mechanisms of fast ions suggest interaction of these with plasmas waves, e.g. energetic particle modes or interaction of fast ions with magnetohydrodynamic normal modes such as toroidal Alfvén eigenmodes [7-14]. The predicted consequences of such effects can be tested against the experimental data obtained by CTS. Additionally, a large portion of the fast ions in ITER will be fusion born alphas. While fast ions generated by auxiliary heating have strongly anisotropic distributions, fusion born alphas have no preferred direction at birth though they become anisotropic due to finite orbit width effects and loss and redistribution. It will therefore be of interest to map the phase space distribution of fast ions in ITER. Even directional resolution will be feasible by CTS measurements. However, one cannot tell from the spectrum how the fast ions have been generated or which species the fast ions belong to.

The goal of the present study is to elucidate which fast ion species makes the most significant contribution to the total CTS signal for a steady-state ITER burning plasma scenario. It is clear that fusion alphas generate a strong CTS signal. However, the question arises whether fusion alpha physics can be observed by CTS even under presence of $1 \mathrm{MeV}$ beam deuterons in the case of NBI or fast tritons or ${ }^{3} \mathrm{He}$ in the multi $\mathrm{MeV}$ range in the case of ICRH. It is conceivable that fast ions generated by these auxiliary heating schemes may overshadow the fusion alphas in the CTS signal. To answer this question, we determine the expected CTS signals originating from fusion alphas as well as from fast ions generated by auxiliary heating and compare their relative sizes. The energetic particle populations due to two off-axis ICRH scenarios and NBI heating have been computed with the PION and ASCOT codes, respectively [15-18]. A fully electromagnetic low temperature kinetic model of CTS is applied to find the corresponding CTS signals for these computed fast ion populations $[19,20]$. In the spectrum of the scattered radiation, these contributions lie in frequency bands beyond the bulk ion feature as discussed in Section 3.1. The calculated contributions of various species to the CTS signal will then facilitate the interpretation of the measurements. It has been found previously that the fast deuterons originating from NBI create a small bump in the CTS spectrum of the near parallel velocity component with respect to the magnetic field for frequency upshifts within a limited frequency band in a limited region 
in space [21]. We compute here the impact of ICRH on alpha particle measurements in ITER and corroborate and extend the previous study of beam ions.

In Section 2, the suggested ITER CTS system is briefly described. The relevant definitions and modelling aspects regarding CTS and the ion distributions including computational methods are outlined in Section 3. The impact of the auxiliary heating schemes on fast ion measurements for near perpendicular velocity resolution and near parallel velocity resolution are discussed in Section 4, and conclusions are drawn in Section 5 .

\section{Design of the CTS System for ITER}

The proposed fast ion CTS diagnostic for ITER consists of two separate systems [22-25]. One system is designed to resolve near perpendicular velocity components with respect to the magnetic field whereas the other resolves near parallel velocity components. The geometries of the two systems are depicted in Figures 1(a) and 2(a), respectively. Each system has one probe beam, each entering the plasma on the low field side (LFS), and several receiver beams out of which some examples are plotted in these figures. The term "receiver beam" refers to scattered radiation accepted by the antenna optics. The displayed beams have been found by ray tracing taking into account the geometry of ITER. The beam paths are curved due to density gradients in the plasma. Additionally, the beam curvatures in Figures 1(a) and 2(a) have to be viewed with caution as they show projections of three-dimensional, curved beam paths (although distortion effects are small). The red line in Figure 2(a) marks the magnetic axis. The near perpendicular velocity components of fast ions can be found by measuring back-scattered radiation received with an antenna on the LFS as sketched in Figure 1(b) and explained further in Section 3.1. The LFS antenna is currently being designed to fit into ITER port plug \#12. However, to resolve near parallel velocities, it will be necessary to collect forward-scattered radiation on the high field side (HFS). This requires a receiver which is designed to fit behind a blanket module from which some blanket material will have to be cut out to accommodate the antenna. The HFS receiver is currently not included in the diagnostic system of ITER. Both CTS systems are proposed to be installed in the same ITER sector. Both systems launch electromagnetic waves in Xmode at $60 \mathrm{GHz}$, generated by $1 \mathrm{MW}$ gyrotrons, into the plasma where the waves interact with fluctuations in the plasma in scattering processes described in Section 3.1. With the proposed ITER CTS system, it will be possible to measure time-resolved fast ion velocity distributions in several measurement volumes simultaneously. The measurement volumes are situated at the intersection of the probe beam and each receiver beam. The CTS signal emanates from the scattering volume whose size, and therewith also the spatial resolution, is determined by the beam dimensions and the angle they span. The resolution is $40 \mathrm{~ms}$ in time, $a / 10$ in space (where $a$ is the minor radius), and the velocity distribution is resolved in at least 16 velocity nodes with adequate accuracy. These parameters satisfy the ITER measurement requirements for 

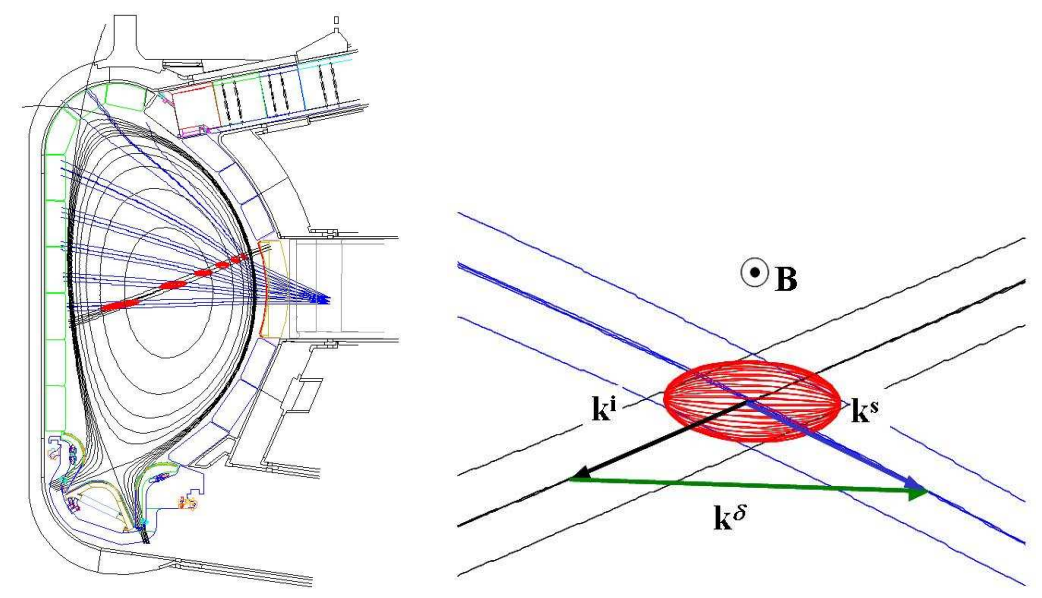

(a) One probe beam (b) LFS scattering geometry containing (black) crossing several the probe beam with wavenumber $\mathbf{k}^{i}$ receiver beams (blue) in (black) and a receiver beam with ITER poloidal plane wavenumber $\mathbf{k}^{s}$ (blue)

Figure 1. Sketch of the LFS CTS system for ITER observing near perpendicular to the magnetic field

fusion alpha diagnostic [26]. Each system will contain 7-10 spatial channels. The sizes of the scattering volumes vary as function of location in the plasma due to variations in local beam sizes and scattering angles: Most scattering volumes have sizes of about $20 \mathrm{~cm}$ while the largest scattering volume has a size of about $50 \mathrm{~cm}$. The angles with respect to the magnetic field for resolution of near perpendicular directions and near parallel directions also depend on the position of the scattering volume and are $100-110^{\circ}$ and $0-20^{\circ}$, respectively. One may note that angles very close to $90^{\circ}$ are avoided: In this range, the fast magnetosonic wave causes a very large spectral response at the fluctuation frequency which satisfies the dispersion relation [20]. As movable parts near the plasma are avoided in the design of the CTS systems for ITER, the spatial channels are fixed in space (although an option of remote steering of the probe is being considered).

\section{Modelling Methods and Assumptions}

\subsection{Collective Thomson Scattering}

CTS refers to scattering of electromagnetic radiation from fluctuations caused by collective motion of electrons. The wavelength of the fluctuations must then be larger than the Debye length $\lambda_{D}$ as expressed by Salpeter's condition $\left(k^{\delta} \lambda_{D}\right)^{-1}>1$ where $k^{\delta}$ is

the magnitude of the fluctuation wave vector $\mathbf{k}^{\delta}$. At wavelengths smaller than the Debye length, the electron motion is uncorrelated and the technique is then called incoherent Thomson scattering which diagnoses electrons. The resolved fluctuation wave vector in the scattering volume is given by the matching conditions $\left(\mathbf{k}^{\delta}, \omega^{\delta}\right)=\left(\mathbf{k}^{s}-\mathbf{k}^{i}, \omega^{s}-\omega^{i}\right)$ involving the wave vectors and frequencies of the incident radiation in the probe 


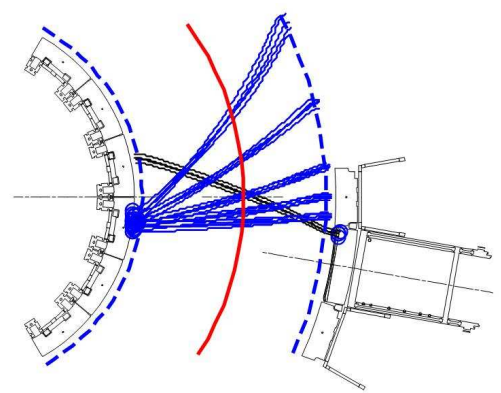

(a) One probe beam (black) cross- (b) HFS scattering geometry containing the ing several receiver beams (blue) probe beam with wavenumber $\mathbf{k}^{i}$ (black) and a in ITER toroidal plane

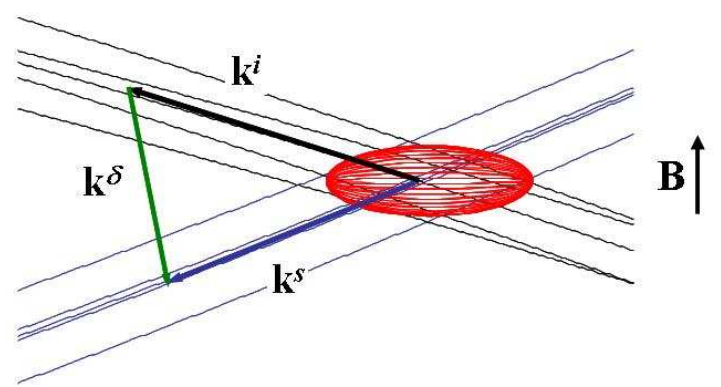

receiver beam with wavenumber $\mathbf{k}^{s}$ (blue)

Figure 2. Sketch of the HFS CTS system for ITER observing near parallel to the magnetic field

beam $\left(\mathbf{k}^{i}, \omega^{i}\right)$ and of the scattered radiation in the receiver beam $\left(\mathbf{k}^{s}, \omega^{s}\right)$, respectively. Appealing to the Doppler shift picture, the frequency shift of the scattered radiation, $\omega^{\delta}$, can be approximately related to the ion velocity by $\omega^{\delta}=\mathbf{v}_{i o n} \cdot \mathbf{k}^{\delta}$, i.e. it depends on the projection of $\mathbf{v}_{i o n}$ onto $\mathbf{k}^{\delta}$ and hence not only on their magnitudes but also the angle between them. The locations of the scattering volumes are here described by the standard ITER coordinates R and Z, neglecting the toroidal coordinate. Two angles are most relevant when describing the scattering geometry: The angle $\phi$ between the magnetic field vector $\mathbf{B}$ and $\mathbf{k}^{\delta}$ and the scattering angle $\theta$ between the probe and receiver beams. It follows from the described projection above that in CTS one resolves the projection $g$ of the full velocity distribution function $f$ along the direction of $\mathbf{k}^{\delta}$ in the scattering volume, expressed by ( $\delta$ is here the Dirac $\delta$-function):

$$
g(u)=\int d^{3} \mathbf{v} f \delta\left(\frac{\mathbf{v} \cdot \mathbf{k}^{\delta}}{k^{\delta}}-u\right)
$$

Only a tiny fraction of the emitted gyrotron power $P^{i}$ is scattered and detected by the receiver. The spectral power density of scattered radiation $\frac{\partial P^{s}}{\partial \omega^{s}}$ is proportional to the scattering function $\Sigma$. The scattering function is derived from a collisionless kinetic description $[19,20]$. It accounts for the spectral variation of microscopic fluctuations in the plasma and therewith also in the received scattered power density. The scattering function is fully electromagnetic and depends on fluctuations in electron density $\tilde{n}$, the electric field $\tilde{\mathbf{E}}$, the magnetic field $\tilde{\mathbf{B}}$, and the current $\tilde{\mathbf{j}}$. The separate contributions of the electrons and several ion species to the scattering function can be found and compared, and the relative contributions to the expected CTS signal can be determined for the entire spectrum. Hence the results in this study are presented in terms of the scattering function.

\subsection{Fusion Alphas and Plasma Parameters}

To determine the spectrum of the scattered radiation, one must make several assumptions regarding the bulk plasma and the distribution functions of the species 
present in the plasma. The densities of the bulk ion species, impurities, and the electrons at the respective measurement location are assumed to be given by the steady-state ITER plasma equilibrium, also called scenario 4, characterized by an internal transport barrier and reversed shear [27]. The parameters for this ITER scenario were taken from simulations with the ASTRA code, e.g. [28]. Additionally, scenario 2 has been investigated, and the impact of fast ions due to auxiliary heating has been found to be smaller compared to that using scenario 4 . As we seek to find situations with the largest possible impact of fast ions due to auxiliary heating, we select here scenario 4 as baseline. The baseline average concentrations of the plasma species are in $n_{i} / n_{e}$ : Deuterium $40 \%$, tritium $40 \%$, alphas $4 \%,{ }^{3} \mathrm{He} 1 \%$, beryllium $2 \%$, and argon $0.1 \%$. The effective charge $Z_{\text {eff }}$ is kept at 1.66 throughout this study. Bulk plasma species are assumed to have Maxwellian distribution functions. For the fusion alphas, we use an isotropic classical slowing down distribution since the anisotropy in the alphas is expected to be small [21]. The 4D distribution functions in two spatial and two velocity coordinates of the other fast ion populations have been calculated with the computational techniques described in Sections 3.3 and 3.4 where also additional modelling parameters are provided. The assumed plasma equilibria are not necessarily consistent with the presented auxiliary heating simulations: The distribution of NBI and ICRH power may vary from the assumptions in the original transport scenario which assumes a total of 40 MW auxiliary heating power [28]. Our assumed ICRH power is $20 \mathrm{MW}$, and the total beam power is $33 \mathrm{MW}$ as is currently foreseen in ITER, but we do not apply both heating scenarios simultaneously in our study. These uncertainties limit the conclusions that can be drawn. Moreover, detailed designs of several hardware components of the auxiliary heating systems are still not available.

\subsection{Fast ions from ICRH}

The PION code was used for the simulations of ICRH scenarios reported here [15,16,29]. The code is time dependent and combines a calculation of the wave power deposition with a kinetic solver for the distribution functions of the resonating ions. It is based on simplified modelling which makes it fast and suitable for routine analysis of ICRH scenarios. At the same time, it seeks to treat the two processes of power deposition and evolution of the distribution function of the resonating ions in an internally self-consistent way. Most importantly, the absorption strength in the power deposition model is made consistent with that of the Fokker-Planck calculation of the distribution functions by evaluating corrections to the dielectric tensor contributions of the resonating ions at the end of every simulation time step; the modified dielectric tensor is then used in the wave power deposition calculation for the next time step; the process is repeated until the end of the calculations. In the scenarios analyzed here, this feature is important since the power partition between the species evolves in time. In particular, since absorption at cyclotron harmonics, $\omega=n \omega_{c i}, n \geq 2$, is a finite Larmor radius (FLR) effect, the absorption strength can vary significantly as a non- 


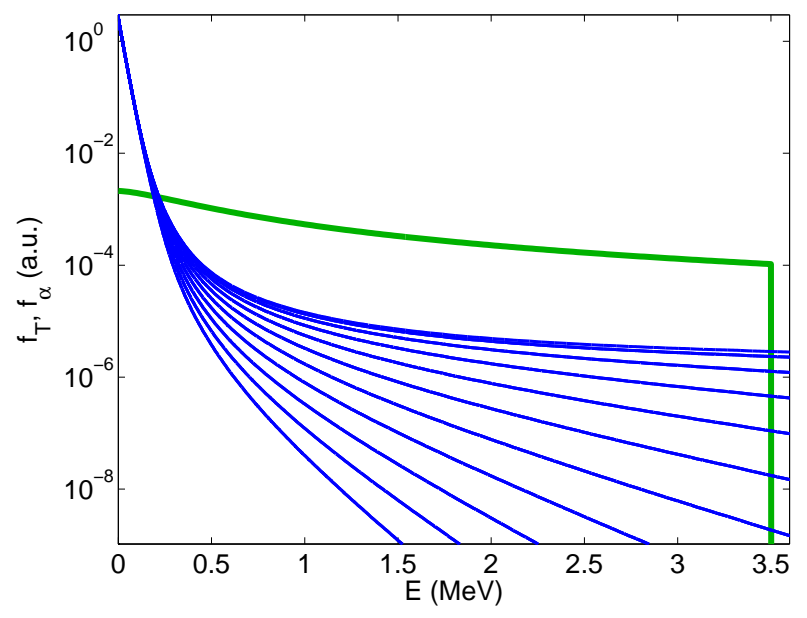

Figure 3. Tritium distribution function for pure second harmonic tritium heating for $R=6.85 \mathrm{~m}$ and $Z=0.77 \mathrm{~m}$ compared to a classical slowdown distribution for alphas; thick green line - alphas, thin blue lines - tritium at various equally spaced pitch angles from $0^{\circ}$ (bottom curve) to $90^{\circ}$ (top curve); the corresponding CTS signal is shown in Figure 6

thermal tail develops on the distribution function of the resonating ions. These finite orbit width effects are important for ICRH tuned to the second harmonic resonance in the center of the plasma. However, for providing bulk heating it is better to move the cyclotron resonance somewhat to the LFS, especially for obtaining a better ion heating fraction [30]. We opted here for the modelling of such scenarios: The magnetic field in the center is the nominal one for ITER, $5.3 \mathrm{~T}$, and the ICRH frequency is $50 \mathrm{MHz}$, placing the second harmonic cyclotron resonance of tritons and fundamental resonance of ${ }^{3} \mathrm{He}$ ions at around a third of the minor radius on the LFS of the magnetic axis. As a result of such a shift, the ICRH power is absorbed over a greater volume, reducing the power density. The orbit width is then reduced since the current enclosed by a flux surface at the resonance layer is higher. For this scenario, finite orbit width effects do not play a significant role. The importance of finite orbit width effects for ITER scenarios has also been assessed by PION simulations, leading to the conclusion that they may be important if the resonance is tuned to the center of the plasma but only play a minor role in the scenarios investigated here.

In the standard version of PION, only the pitch angle averaged distribution function is calculated together with an estimate of the averaged square velocity of the resonating ions (important for assessing the Doppler broadening of the cyclotron resonance). Moreover, finite orbit width effects are taken into account by assuming that the nonthermal resonating ions have turning points close to the cyclotron resonance, i.e. where the cyclotron frequency of a resonating ion is close to the wave frequency at the turning point, $\omega \approx n \omega_{c i}(R)$ ( $n=1$ for interaction at the fundamental frequency and $n=2$ for second harmonic interaction). Collision coefficients are averaged over the resulting orbits, and the orbits are also used to redistribute fast ion pressures, power transfer via collisions to bulk plasma ions and electrons etc. In the simulations presented here, it 
is important to assess the pitch angle dependence of the distribution functions of the resonating ions. For this purpose, the standard PION version has been augmented by a module providing a model for the pitch angle distribution in the small banana width limit which is similar to that found in e.g. Ref. [31]. This model should provide acceptable results for cases where finite orbit width effects are not expected to be important. The distribution functions discussed in this study were evaluated with a finite difference method on 40 flux surfaces equally spaced in the flux coordinate given by the square root of the poloidal flux. This corresponds also fairly closely to a uniform grid spacing in the normalized minor radius.

An example distribution function, computed with the PION code, is plotted in Figure 3 for $R=6.85 \mathrm{~m}$ and $Z=0.77 \mathrm{~m}$. The anisotropy of the particle phase space density in parallel and perpendicular velocities is evident. This anisotropy arises since particles are accelerated mostly in the perpendicular direction by ICRH.

\subsection{Fast ions from NBI}

In this work, the EDA2001 NBI design is used [32]. The two NB injectors each deliver 16.5 MW of power carried by a co-injected deuterium beam with the energy of $1 \mathrm{MeV}$ per particle. The tangency radius is $R_{\text {tang }}=5.295 \mathrm{~m}$ for both beams, but the beam sources can be tilted horizontally to heat the plasma on-axis or off-axis. In this work, the total power of $33 \mathrm{MW}$ was taken half on-axis, half off-axis. The beam ion distribution functions were computed with the test-particle Monte Carlo code ASCOT $[17,18]$ that follows the guiding center orbits of charged particles in realistic 3D tokamak geometries including the toroidal ripple.

The neutral beam ion distribution is generated by modelling the individual beamlets from the source grid. In this work, beam ion initialization supplied by the PENCIL code [33] was used. A large number of test particles $\left(10^{5}\right)$ was simulated. Interaction between the beam particles and the steady-state Maxwellian background plasma is modelled between orbit-integration time steps using binomially distributed Monte Carlo operators. The Coulomb collision operators are derived from the Lorentz and FokkerPlanck collision operators using Rosenbluth potentials [34,35]. The test particles were simulated until they either hit a material surface or were slowed down below $100 \mathrm{keV}$.

Realistic temperature and density profiles of the ITER steady-state equilibrium (Scenario 4) were used as the plasma background for the simulations. The magnetic field was assumed to be axisymmetric. During the simulation, the parallel and perpendicular velocities of the particles were recorded, along with their location in the poloidal crosssection, yielding a four-dimensional distribution. Figure 4 displays the resulting beam ion velocity distribution at $(\mathrm{R}, \mathrm{Z})=(6.12 \mathrm{~m}, 0.64 \mathrm{~m})$, and Figure 5 shows the spatial profile of the source term for the test particles. The dimensions of the computed fourdimensional slowing down distribution are as follows: Spatially 30 nodes in R-direction and 50 nodes in Z-direction, and in the velocity space 40 by 20 nodes in parallel and perpendicular velocities, respectively. 


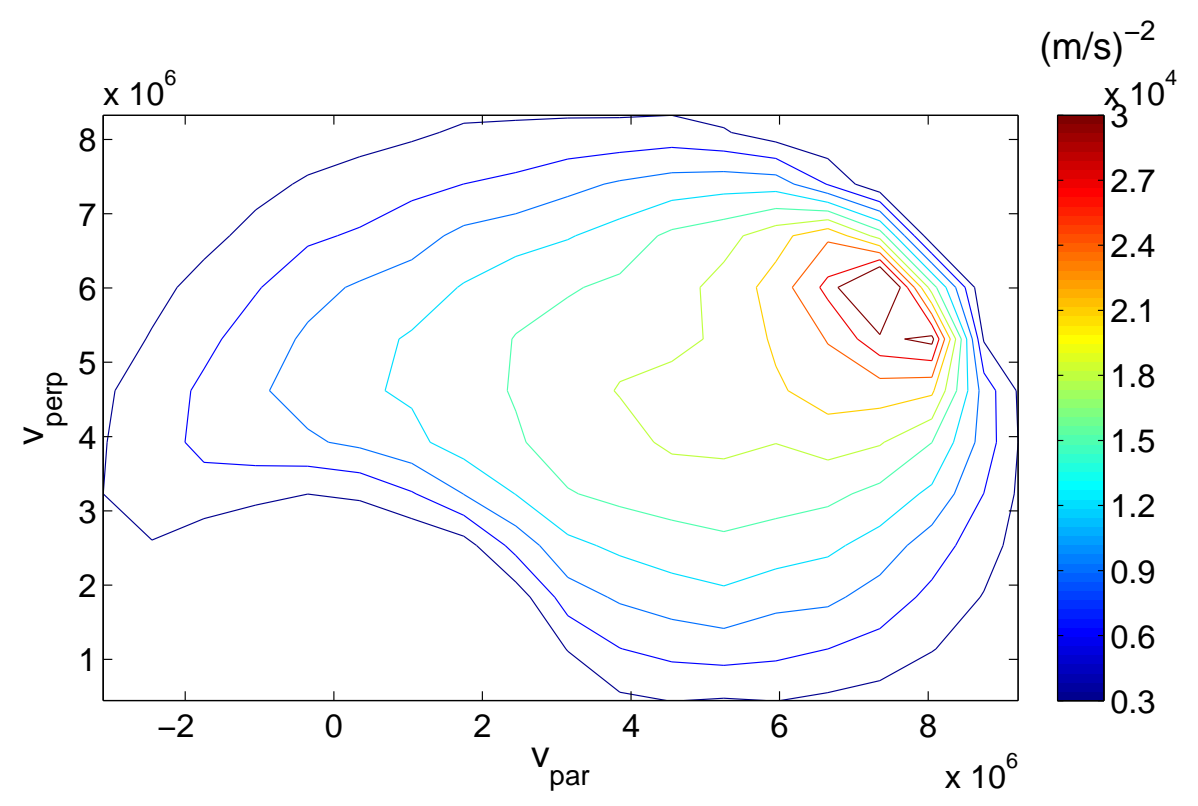

Figure 4. Beam ion distribution function for $R=6.12 \mathrm{~m}$ and $Z=0.64 \mathrm{~m}$; isolines are equally spaced with a difference of $0.3(\mathrm{~m} / \mathrm{s})^{-} 2$ as indicated in the legend; the corresponding CTS signal is shown in Figure 9

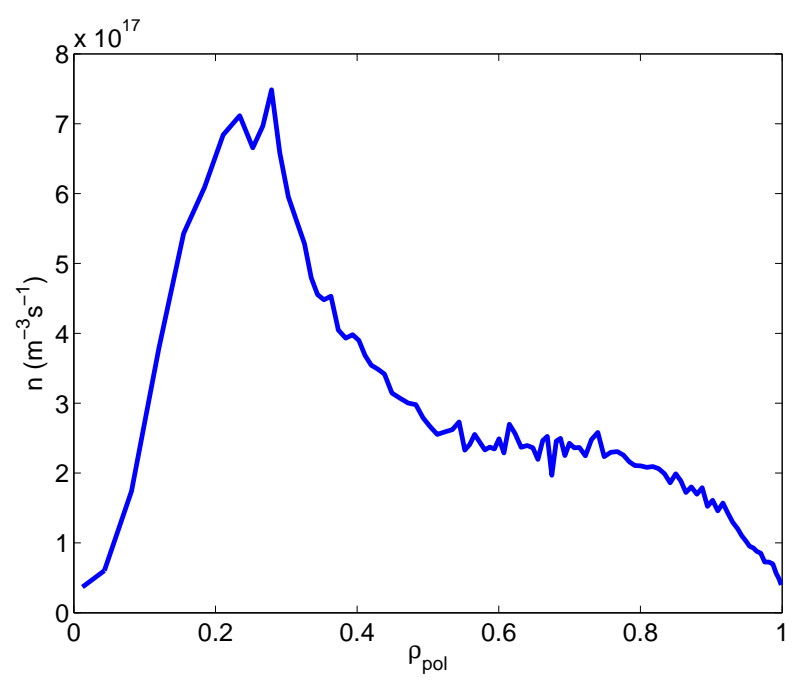

Figure 5. Spatial profile of the beam ion slowing down distribution at birth time

\section{Results}

\subsection{Resolution of Near Perpendicular Velocities by the LFS CTS System}

The near perpendicular velocity components of the fast ion population can be resolved with probe and receivers on the LFS as depicted in Figure 1(a). We investigate here two heating scenarios for ICRH as described in Section 3.3: Heating of tritium at the second harmonic and minority heating of ${ }^{3} \mathrm{He}$ at the fundamental resonance. In both cases, the resonance at $50 \mathrm{MHz}$ lies off-axis on the LFS. Additionally, the fast deuteron 
population from NBI is investigated.

We seek to find the scattering volumes and geometries in the plasma for which the computed CTS signal component due to the auxiliary heating compared to the alpha CTS signal component is maximized. More than one hundred measurement volumes have been computed by ray tracing. All such beams and scattering volumes are compatible with the geometry constraints imposed by the ITER machine. In the planned ITER CTS design, at most 10 scattering volumes are foreseen for each system. Thus we search for the maximum signal with a much finer scanning step size compared to the planned ITER CTS experiment: The coordinates of two such hypothetical neighboring scattering volumes differ by only $2-3 \mathrm{~cm}$, ensuring that a good approximation to the location with maximum CTS signal fraction due to auxiliary heating is found. This resolution is higher than what is provided by either the ICRH fast ion population (given on 40 flux surfaces) or the beam ion population (30 nodes in R-direction and 50 nodes in Z-direction). The CTS signals are computed for the center points of these measurement volumes, neglecting their spatial extent. The maximum signal for the ICRH pure tritium at the second harmonic resonance scenario is situated at $R=6.85 \mathrm{~m}$ and $Z=0.77 \mathrm{~m}$. The distribution function for these coordinates is plotted in Figure 3 which was already mentioned in Section 3.3.

Figure 6 reveals the corresponding CTS signal at this location resolved in nearly perpendicular direction $\left(\phi=\angle\left(\mathbf{k}^{\delta}, \mathbf{B}\right)=101^{\circ}\right)$. The ordinate measures the magnitude of the scattering function as a function of frequency shift $\nu^{\delta}$ from the probe beam frequency $\nu^{i}$. The total signal in Figure 6 is the sum of the individual contributions of electrons and the present ion species. The alpha feature dominates the spectrum for frequency shifts beyond $\sim 1 \mathrm{GHz}$ up to $\sim 4 \mathrm{GHz}$ (the outermost part of the wing corresponding to the alpha birth energies). The CTS signals due to deuterium and the impurities beryllium and argon are lumped as bulk ions with thermal velocities, leading to a signal component in the inner frequency band up to $\sim 1 \mathrm{GHz}$ on each side which towers over the much lower alpha feature. The tritium feature is singled out from the bulk ions as the tritium distribution function develops highly energetic tails on each side even at higher frequency shifts than found for alphas. Energy absorbtion in ICRH at the second harmonic increases with the Larmor radius, and since the Larmor radius itself becomes larger with temperature, it is clear that exceedingly energetic tails form in this heating scenario. However, for the off-axis heating scenario we investigate here, the power density is rather low compared to a corresponding on-axis heating scenario since the volume in which the power is absorbed is large. The tritium feature is even below the electron feature, and no interference in the alpha measurement due to tritium is therefore expected. We point out that this does not imply that fast ions from ICRH cannot be measured in the pre-burn phase of ITER since the plasma background parameters are different. Additional simulations of ITER plasmas in the pre-burn phase will be required to address this.

It can be beneficial to add small amounts of ${ }^{3} \mathrm{He}$ to the plasma in a minority heating scheme. The fundamental resonance layer of ${ }^{3} \mathrm{He}$ and the second harmonic of tritium 


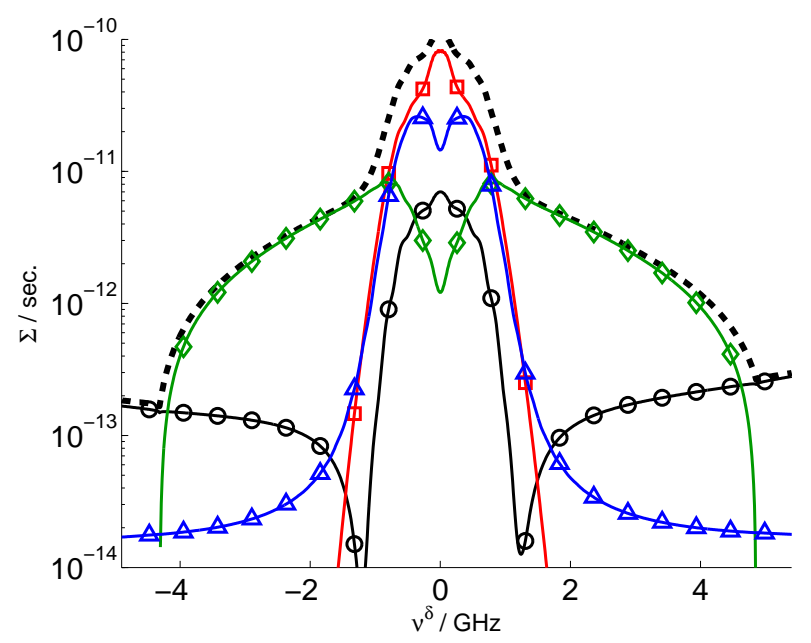

Figure 6. Scattering function for pure second harmonic tritium heating; selection of near perpendicular velocities; scattering parameters: $R=6.85 \mathrm{~m}, Z=0.77 \mathrm{~m}$, $\phi=101^{\circ}, \theta=156^{\circ} ; \diamond$-alphas, $\triangle$-tritons, $\square$-bulk ions, o-electrons, ----total

coincide. The slowing down time is proportional to the mass and the inverse square of the charge, implying that ${ }^{3} \mathrm{He}$ slows down four times faster than tritium. For the same absorbed power, the high energy tail of ${ }^{3} \mathrm{He}$ will therefore be less populated compared to the tritium tail. Since the critical velocities for ${ }^{3} \mathrm{He}$ and tritium are identical, a larger fraction of the tail falls below the critical velocity for ${ }^{3} \mathrm{He}$, favoring ion heating rather than electron heating. Heating at the fundamental resonance is not strongly dependent on FLR effects compared to second harmonic heating, and the heating is therefore less dependent on the velocities of the resonating ions. One could therefore think that the tail formation is not as pronounced. Nevertheless, a very energetic tail develops due to the fact that the power per resonating particle is high for a minority species. As a result, a fairly strong contribution to the CTS signal can be observed in Figure 7, reaching even above the electron feature. Here, a ${ }^{3} \mathrm{He}$ population at a concentration of $2 \%$ is included as well as the species from Figure 6. This concentration leads to relatively populated tails compared to other ${ }^{3} \mathrm{He}$ concentrations: We find the largest CTS signal fraction for $2-3 \%{ }^{3} \mathrm{He}$ concentration for this heating power. The ${ }^{3} \mathrm{He}$ feature reaches up to ordinates of $10-20 \%$ of the alpha feature in a narrow region close to the resonance layer. We are guided in our judgement of the importance of other signal contributions by the ITER measurement requirements which demand an accuracy of $20 \%$ for fusion alpha diagnostics [26]. In this perspective, the CTS signal in the outer frequency bands contains a non-negligible part due to the minority species. The CTS signal due to ${ }^{3} \mathrm{He}$ is larger than the tritium CTS signal for the corresponding pure second harmonic tritium heating scenario discussed above. Part of the reason is that CTS signals are proportional to the square of the charge and a fast ${ }^{3} \mathrm{He}$ ion therefore generates four times as much scattering as a fast triton proceeding at the same velocity. Secondly, owing to differences in power deposition profiles on the tritons and the ${ }^{3} \mathrm{He}$ ions, the volume with the maximum scattering signal contribution lies slightly to the HFS of that 


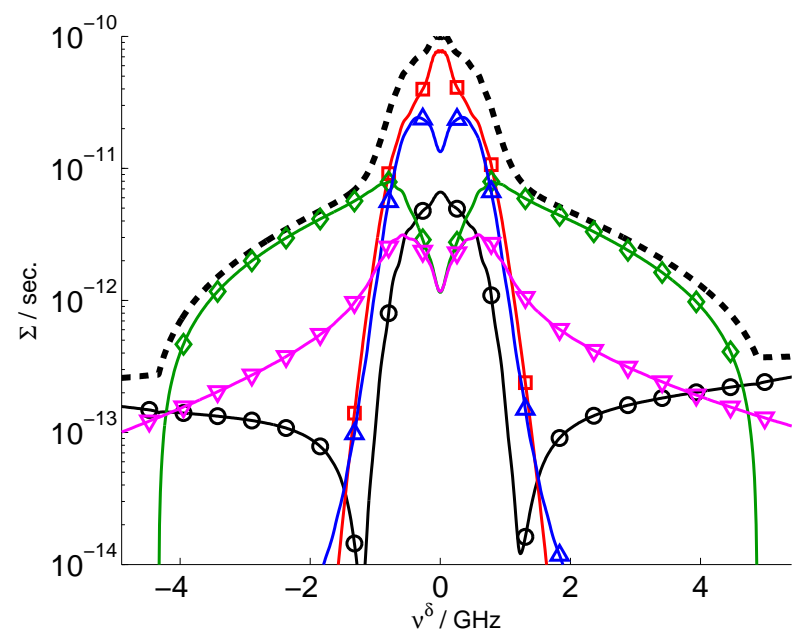

Figure 7. Scattering function for ${ }^{3} \mathrm{He}$ minority heating with $2 \%{ }^{3} \mathrm{He}$; selection of near perpendicular velocities; scattering parameters: $R=6.76 \mathrm{~m}, Z=0.76 \mathrm{~m}, \phi=101^{\circ}$, $\theta=157^{\circ} ; \diamond$-alphas, $\triangle$-tritons, $\nabla^{-}{ }^{3} \mathrm{He}, \square$-bulk ions, o-electrons, ----total

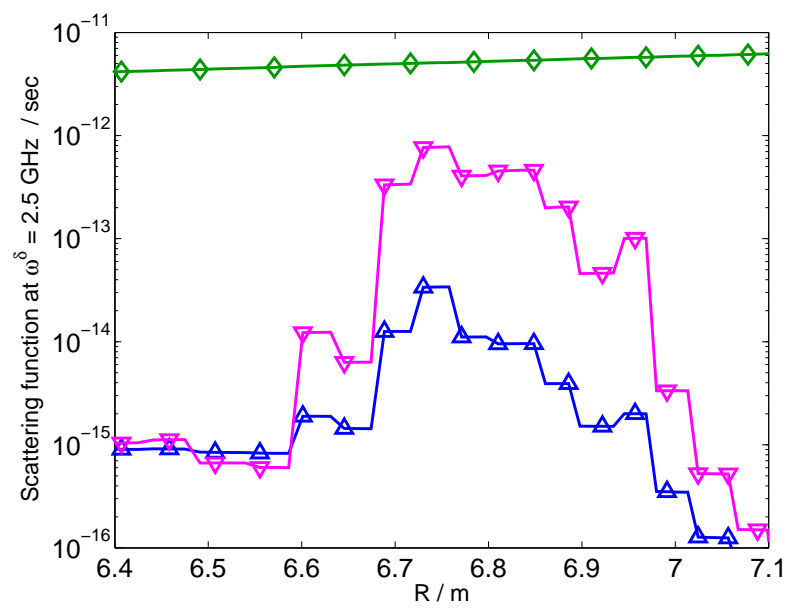

Figure 8. Scattering function at $\omega^{\delta}=2.5 \mathrm{GHz}$ for various scattering volumes with different $R ; \diamond$-alphas, $\triangle$-tritons, $\nabla^{-}{ }^{3} \mathrm{He}$

discussed earlier, leading to a larger volumetric heating rate for the same power. The tritium distribution at this position has a less energetic tail, resulting in a much lower signal compared to before.

Figure 8 shows the expected CTS signal contributions at one representative frequency, $\omega^{\delta}=2.5 \mathrm{GHz}$, as a function of radial coordinate for the ICRH scenario with the ${ }^{3} \mathrm{He}$ minority population. The width of the region with strong CTS signal component from the ${ }^{3} \mathrm{He}$ is rather narrow in space, $\sim 0.2-0.3 \mathrm{~m}$. This size is similar to the size of a typical scattering volume. This indicates that spatial variations in the CTS signal strength may be important if only part of the scattering volume lies in the resonance layer, since we assume a constant CTS signal throughout the scattering volume. This assumption may lead to an overestimation of the signal contributions from 


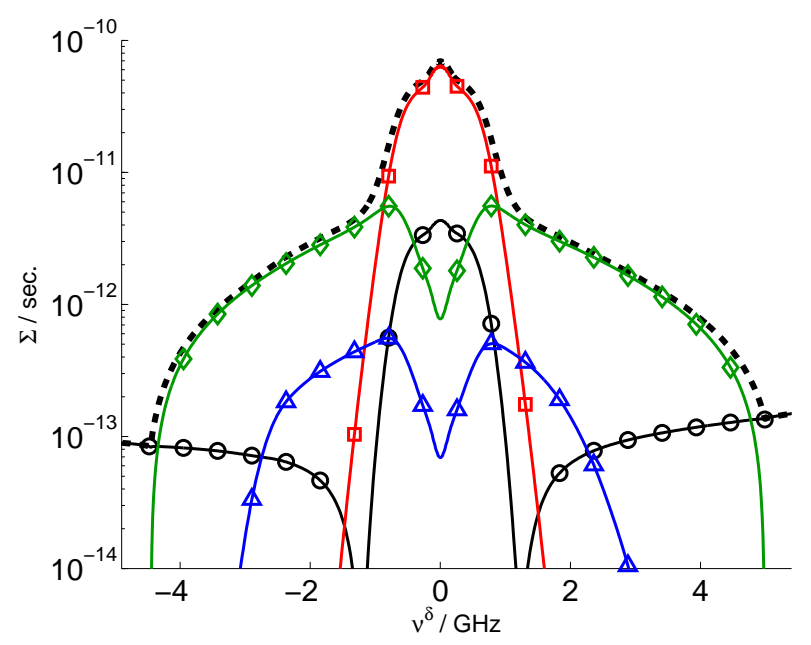

Figure 9. Scattering function for location in the NBI beam path; selection of near perpendicular velocities; scattering parameters: $R=6.12 \mathrm{~m}, Z=0.64 \mathrm{~m}, \phi=101^{\circ}$, $\theta=162^{\circ} ; \diamond$-alphas, $\triangle$-beam ions, $\square$-bulk ions, O-electrons, ----total

the resonating species if the scattering volume extends further than the resonance layer. Therefore, we can be sure that we do not miss any region with non-negligible CTS signal fraction due to the auxiliary heating. The width of the region with strong CTS signal is similar for the pure second harmonic tritium heating scenario whereas beam ions (to be discussed next) contribute a strong CTS signal component over a wider region $(\sim 1.4 \mathrm{~m})$ which is much larger than a typical scattering volume.

Fast ions originating from NBI may also be detected by the LFS CTS system as this population has a perpendicular velocity component due to the given injection geometry. In fact, as Figure 4 demonstrates, the perpendicular velocities are not much smaller than the parallel velocities as the injection pitch angle cosines lie between 0.5 and 0.7 for a large share of the beam ions. The velocity distribution in this figure results in the largest beam ion CTS signal compared to the alpha signal in the scattering volume given by $R=6.12 \mathrm{~m}, Z=0.64 \mathrm{~m}, \phi=101^{\circ}$, and $\theta=162^{\circ}$. The calculated spectra for these parameters are presented in Figure 9. The nomenclature changes slightly for this figure: Bulk ions here contain bulk deuterium, tritium, and the impurities argon, beryllium, and ${ }^{3} \mathrm{He}$. However, the beam ions are singled out and not included in the bulk. The contribution to the CTS signal due to the beam ions is about an order of magnitude smaller than the contribution due to fusion alphas for both upshifted and downshifted frequencies. Thus, the observed spectra of the perpendicular velocity components will not be significantly interfered by the beam ions in the entire frequency band for all spatial locations.

\subsection{Resolution of Near Parallel Velocities by the HFS CTS System}

Near parallel directions can be selected with a receiver on the HFS in the proposed CTS system design as Figure 2(a) illustrates. The fast ions due to ICRH have larger 


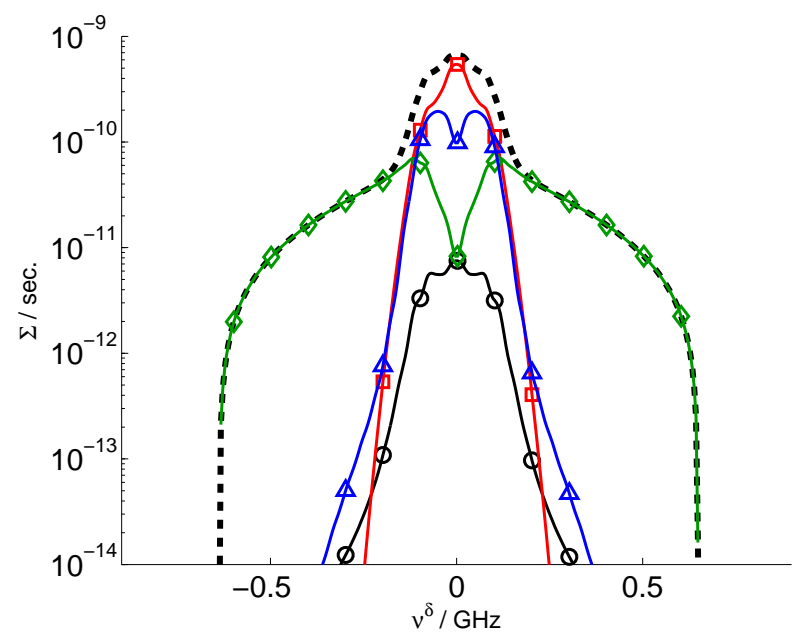

Figure 10. Scattering function for pure second harmonic tritium ICRH; selection of near parallel velocities; scattering parameters: $R=6.86 \mathrm{~m}, Z=0.59 \mathrm{~m}, \phi=2^{\circ}$, $\theta=16^{\circ} ; \diamond$-alphas, $\triangle$-tritons, $\square$-bulk ions, o-electrons, ----total

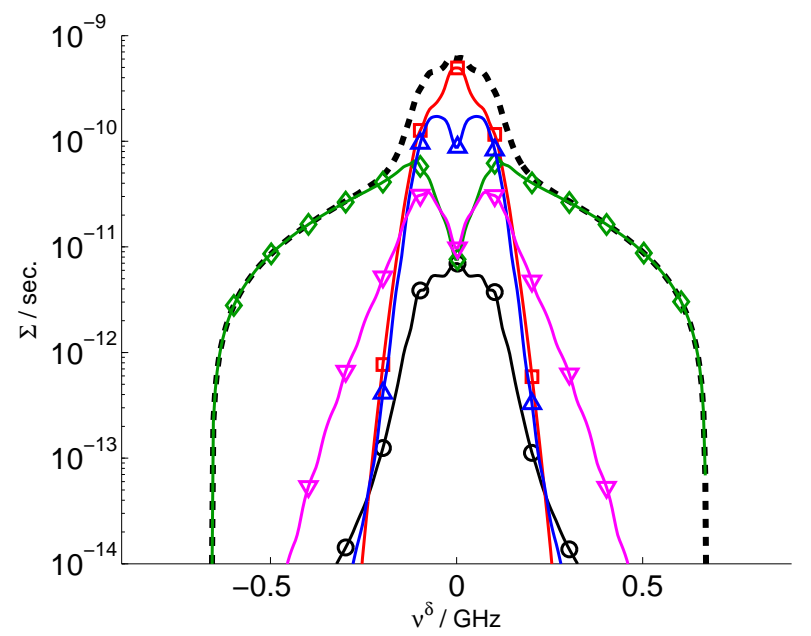

Figure 11. Scattering function for minority ${ }^{3} \mathrm{He}$ ICRH; selection of near parallel velocities; scattering parameters: $R=6.76 \mathrm{~m}, Z=0.59 \mathrm{~m}, \phi=2^{\circ}, \theta=16^{\circ} ; \diamond$-alphas, $\triangle$-tritons, $\nabla^{-}{ }^{3} \mathrm{He}, \square$-bulk ions, o-electrons, ----total

velocity components in the near perpendicular direction and are therefore not expected to result in a strong signal in the HFS receiver. The corresponding CTS signals for the pure tritium heating and the minority heating scenarios are depicted in Figures 10 and 11, respectively. Each scattering volume is less than $0.2 \mathrm{~m}$ (mostly in Z direction) away from the corresponding scattering volume for the LFS CTS system. The distribution functions are similar to corresponding distributions functions from the LFS system discussed previously due to this proximity. The CTS signals of these fast ion populations are small compared to the alpha signals. The suggested HFS CTS system would therefore resolve near parallel velocities of alphas not much perturbed by energetic ions due to ICRH. 


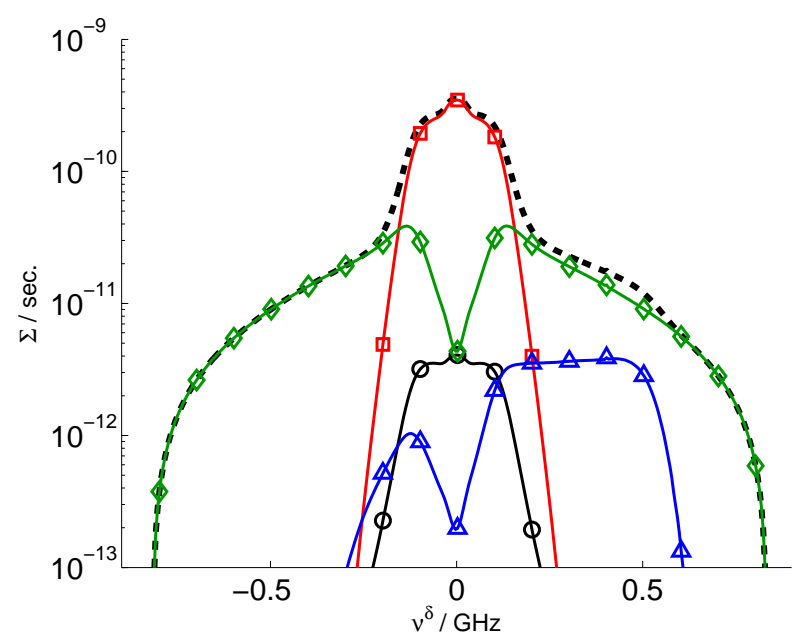

Figure 12. Scattering function for location in the NBI beam path; selection of near parallel velocities; scattering parameters: $R=6.18 \mathrm{~m}, Z=0.59 \mathrm{~m}, \phi=5^{\circ}, \theta=20^{\circ}$; $\diamond$-alphas, $\triangle$-beam ions, $\square$-bulk ions, o-electrons, ----total

However, the fast beam deuterons have a large velocity component parallel to the magnetic field and are therefore detectable by the HFS CTS system. The scattering function for this case is shown in Figure 12 for a location at $R=6.18 \mathrm{~m}$ and $Z=0.59 \mathrm{~m}$ and the angles $\phi=5^{\circ}$ and $\theta=20^{\circ}$. The coordinates are again close to the corresponding coordinates for the LFS system. The beam ion signal has a significant contribution compared to the fusion alpha signal for frequency upshifts in the frequency band from +0.2 to $+0.6 \mathrm{GHz}$ though the contribution is still smaller than the alpha contribution by a factor three. The beam contribution is above $20 \%$ of the alpha signal between the major radii $R=5.70 \mathrm{~m}$ and $R=7.10 \mathrm{~m}$. The results indicate that in this range it may be difficult to draw conclusions about the distribution function of the fusion alphas. However, the beam ions are highly anisotropic (see Figure 4), and they influence the signal only for frequency upshifts in the present geometry, leading to the clear asymmetry of the beam ion feature in Figure 12. For frequency downshifts the beam ion contribution is several orders of magnitude smaller than the fusion alpha contribution and clearly negligible. The fusion alphas can therefore be diagnosed, undisturbed by beam ions, for frequency downshifts, even at the location with the strongest beam ion contribution to the CTS signal. These conclusions are also consistent with previous findings [21].

\section{Conclusions}

The present study indicates that the proposed fast ion CTS diagnostic for ITER can measure the fusion alpha distributions even in the presence of energetic ions due to ICRH or NBI with good accuracy. The fusion alpha CTS signal contribution in the outer frequency bands is indeed more than an order of magnitude larger than contributions from NBI or off-axis ICRH in almost all cases. However, NBI ions may have a significant contribution $(\sim 30 \%)$ detectable by the HFS CTS system resolving 
near parallel directions, but only in a limited region in space and only for frequency upshifts but not downshifts. The CTS signal component due to fast ions accelerated by off-axis ICRH is more than an order of magnitude smaller than the alpha CTS signal component even in near perpendicular direction to be measured by the LFS receiver. However, for the investigated ${ }^{3} \mathrm{He}$ minority heating scheme, the contribution can be $\sim 10-20 \%$, but only close to the resonance layer. Such low CTS signal contributions from auxiliary heating indicate that it will in turn be difficult to observe the fast ions due to auxiliary heating in the presence of alpha particles.

\section{Acknowledgments}

This work, supported by the European Communities under the contract of Association between EURATOM and Risø DTU, CEA, and TEKES, was partly carried out within the framework of the European Fusion Development Agreement. The views and opinions expressed herein do not necessarily reflect those of the European Commission. The computing facilities of the Finnish IT Center for Science (CSC) used in this work are thankfully acknowledged.

\section{References}

[1] Bindslev H, Hoekzema J A, Egedal J, Fessey J A, Hughes T P and Machuzak J S 1999 Phys. Rev. Lett. 83(16) 3206-3209

[2] Bindslev H, Nielsen S K, Porte L, Hoekzema J A, Korsholm S B, Meo F, Michelsen P K, Michelsen S, Oosterbeek J, Tsakadze E L, Westerhof E, Woskow P and the TEXTOR team 2006 Phys. Rev. Lett. 97 205005-1-2005005-4

[3] Bindslev H, Nielsen S K, Porte L, Hoekzema J A, Korsholm S B, Meo F, Michelsen P K, Michelsen S, Oosterbeek J, Tsakadze E L, Westerhof E, Woskow P and the TEXTOR team 2007 Plasma Phys. Control. Fusion 49 B551-B562

[4] Nielsen S K, Bindslev H, Porte L, Hoekzema J A, Korsholm S B, Meo F, Michelsen P K, Michelsen S, Oosterbeek J, Tsakadze E L, Westerhof E, Woskow P and the TEXTOR team 2008 Phys. Rev. E $\mathbf{7 7} 16407$

[5] Heidbrink W and Sadler G 1994 Nucl. Fusion 34535

[6] Jacquinot et al J 1999 Nucl. Fusion 39 2471-2495

[7] Pinches S D, Berk H L, Borba D N, Breizman B N, Briguglio S, Fasoli A, Fogaccia G, Gryaznevich M P, Kiptily V, Mantsinen M J, Sharapov S E, Testa D, Vann R G L, Vlad G and Zonca F 2004 Plasma Phys. Control. Fusion 46 B187-B200

[8] Fasoli A, Gormenzano C, Berk H, Breizman B, Briguglio S, Darrow D, Gorelenkov N, Heidbrink W, Jaun A, Konovalov S, Nazikian R, Noterdaeme J M, Sharapov S, Shinohara K, Testa D, Tobita K, Todo Y, Vlad G and F Z 2007 Nucl. Fusion 47 S264 - S284

[9] Rosenbluth M and Rutherford P 1975 Phys. Rev. Lett. 34 1428-1431

[10] Cheng C and Chance M 1986 Phys. Fluids 29 3695-3701

[11] Zweben S, Budny R, Darrow D, Medley S, Nazikian R, Stratton B, Synakowski E, Taylor G and the TFTR Group 2000 Nucl. Fusion $4091-149$

[12] Heidbrink W 2002 Phys. Plasma 92213 - 2219

[13] Chen L and Zonca F 2007 Nucl. Fusion 47 S727 - S734

[14] Briguglio S, Fogaccia G, Vlad G, Zonca F, Shinohara K, Ishikawa M and Takechi M 2007 Phys. Plasma 14 055904-055904-10 
[15] Eriksson L G, Hellsten T and Willen U 1993 Nucl. Fusion 33 1037-48

[16] Eriksson L G and Hellsten T 1995 Phys. Scripta 5370

[17] Heikkinen J and Sipilä S 1995 Phys. Plasma 23724 - 3733

[18] Heikkinen J, T P Kiviniemi T, Kurki-Suonio T, Peeters A and Sipilä S 2001 J. Comp. Phys. 173 $527-548$

[19] Bindslev H 1993 Plasma Phys. Control. Fusion 35(11) 1615-1640

[20] Bindslev H 1996 J. Atmos. Terr. Phys. 58983

[21] Egedal J, Bindslev H, Budney R and Woskov P 2005 Nucl. Fusion 45 191-200

[22] Bindslev H, Meo F, Tsakadze E, Korsholm S B and Woskov P 2004 Rev. Sci. Instrum. 75(10) 3598-3600

[23] Meo F, Bindslev H, Korsholm S, Tsakadze E, Walker C, Woskov P and Vayakis G 2004 Rev. Sci. Instrum. 75 3585-3588

[24] Tsakadze E, Bindslev H, Korsholm S, Larsen A, Meo F, Michelsen P, Michelsen S, Nielsen A, Nimb S, Lauritzen B, Nonbøl E and Dubois N 2008 Fusion Sci. Tech. $5369-76$

[25] Salewski M, Meo F, Bindslev H, Furtula V, Korsholm S B, Lauritzen B, Leipold F, Michelsen P K, Nielsen S K and Nonbøl E 2008 Rev. Sci. Instrum. 79(10) 10E729

[26] Donne A, AE C, Barnsley R, Bindslev H, Boivin R, Conway G, Fisher R, Giannella R, Hartfuss H, von Hellermann M, Hodgson E, Ingesson L, Itami K, Johnson D, Kawano Y, Kondoh T, Krasilnikov A, Kusama Y, Litnovsky A, Lotte P, Nielsen P, Nishitani T, Orsitto F, Peterson B, Razdobarin G, Sanchez J, Sasao M, Sugie T, Vayakis G, Voitsenya V, Vukolov K, Walker C, Young K and the ITPA Topical Group on Diagnostics 2007 Nucl. Fusion 47 S337 - S384

[27] Shimada M, Campbell D, Mukhovatov V, Fujiwara M, Kirneva N, Lackner K, Nagami M, Pustovitov V, Uckan N, Wesley J, Asakura N, Costley A, Donné A, Doyle E, Fasoli A, Gormezano C, Gribov Y, Gruber O, Hender T, Houlberg W, Ide S, Kamada Y, Leonard A, Lipschultz B, Loarte A, Miyamoto K, Mukhovatov V, Osborne T, Polevoi A and Sips A 2007 Nucl. Fusion 47 S1-S17

[28] Polevoi A 2002 J. Plasma Fusion Res. SERIES 582

[29] Salewski M, Eriksson L G, Bindslev H, Korsholm S B, Leipold F, Meo F, Michelsen P K and Nielsen S K 2008 Proceedings of the 22nd IAEA Fusion Energy Conference (IAEA)

[30] Bergeaud V, Eriksson L G and Start D 2000 Nucl. Fusion 40 35-51

[31] Chang C 1985 Phys. Fluids 283598

[32] IAEA 2002 ITER EDA Documentation Series No.24 Tech. rep.

[33] Challis C, Cordey J, Hamnen H, Stubberfield P, Christinnsen J, Lazzaro E, Muir D, Stork D and Thompson E 1989 Nucl. Fusion 29563

[34] Boozer A and Kuo-Petravic G 1981 Phys. Fluids 24851

[35] Rosenbluth M N, Hazeltine R D and Hinton F L 1972 Phys. Fluids 15 116-140 